\title{
A Study on Problems Faced by the People with Disabilities (PWDs) Using Combined Overlap Block Fuzzy Cognitive Maps (COBFCMS)
}

\author{
A. Praveen Prakash, A. Rajkumar, and N. Jose Parvin Praveena
}

\begin{abstract}
The Imperative reasons for problems faced by the people with Disabilities (PWDs) using combined overlap block fuzzy cognitive maps (COBFCMS) defined by W.B Vasantha Kandasamy is analyzed in this paper. The combined overlap block FCM's defined in this method become effective when the number of concepts can be grouped and are large in numbers. In this paper we analyzed the problems and are large in number. In this paper we analyzed the problem and find out the reasons how it has happened to people. This paper has six sections, first section gives the information about development of fuzzy cognitive maps, second section gives preliminaries of fuzzy cognitive maps, and combined overlap block fuzzy cognitive maps, in section three we explain about the problem faced by PWDs, in section four we explain the method of determining their hidden pattern, in section five, we give the concept of the problem, final section gives the conclusion based on our studies.
\end{abstract}

\section{Index Terms-FCMS, COBFCMS, disablity.}

\section{INTRODUCTION}

Political scientist R. Axelrod [1] introduced cognitive maps for representing social scientific knowledge and describing the methods that are used for decision making in social and political systems. Then B. Kosko [2] enhanced the power of cognitive maps considering fuzzy values for the concepts of the cognitive map and fuzzy degrees of interrelationships between concepts. FCMs can successfully represent knowledge and human experience, introduce concept to represent the essential elements and cause \& effect relationships among the concepts to model the behavior of any system. It is a very convenient, simple and powerful tool, which is used in numerous fields such as social economic and medical etc. The purpose of study is to identify risk groups, The 8 types of disability (by birth not due to disease or accidents or due to war) mentioned in order are as follows: (1) Blindness, (2) Aborted embryo (3) Hump back (lying with the body disposed in a crooked position) (4) Dwarf (5) Dumbness (speech impaired) (6) Deafness, (hearing impaired) (7) No proper development of bones (8) Bewilderment of mind (Not in a position to stand up; these person's mind never grows) (Reference: Pura nanuru poem 28).Now we just mention about how the social and psychological problems of the PWDs were studied and analysed using mathematical and

Manuscript received November 24, 2012; revised January 31, 2013.

A. Praveen Prakash and A. Rajkumar are with the Hindustan University, Padur, chennai 603103, India (e-mail: arajkumar@hindustanuniv.ac.in).

N. Jose Parvin Praveena is with the K.C.G College of Technology, Chennai, India. fuzzy linguistic models. At the outset the authors who are not experts in the technicality of naming the disability may mention the type of disability in a common way, say, as 'blind' instead of "impaired vision" or so; likewise 'deaf' instead of' hearing impaired' and so on. This is by no means to offend or make one feel bad; it is completely the inability of the authors to use proper technical terms. Secondly, the PWDs, most of them physical, is analysed using statistical methods and fuzzy models by the authors. The mental retardation or mental disability is analysed using fuzzy linguistic models. We have categorized the mental disability into five types mainly for our fuzzy linguistic working and they are not in any way medically or technically assigned. The data was collected from interviews from the caretakers of the mentally disabled children / adults, from the homes run by NGO's / charity organization in / near Chennai. Then we used experts. The experts were caretakers or parents or persons closely associated with the mentally disabled people.

The suggestion / conclusions are from the experts and we are not very sure whether it is true or false. For, it is what they feel or they have experienced while taking care of these children/ adults. This only is the first hand experience who have been with these mentally retarded children for over a decade or so. Most of the information gathered is from their practical knowledge. Also when we enquired a few experts in the field some of them said the mental disability cannot be easily detected by birth and lots of research ought to be done in this direction. They also said it was incurable. However our study mainly concentrates on the social and the psychological problems faced by the parents/care givers / relatives of the mentally retarded / PWD, as even today the birth of such children is only thought of as a curse on the family. So the public shun them and their family and they are socially stigmatized. "How to overcome this situation?" is our study. Moreover, the data is an unsupervised one and also there is uncertainty in the concepts. Hence fuzzy tools alone has the capacity to analyze these concepts. Hence it is chosen here.

\section{PRELIMINARIES}

Fuzzy cognitive maps (FCMs) are more applicable when the data in the first place is an unsupervised one. The FCMs work on the opinion of experts. FCMs model the worlds as a collection of classes and causal relation between classes. [3]

Definition 2.1: An FCM is a directed graph with concepts like policies, events etc as nodes and causalities as edges. It represents causal relationship between concepts. 
Definition 2.2: When the nodes of the FCM are fuzzy sets then they are called as fuzzy nodes.

Definition 2.3: FCMs with edge weights or causalities from the set $\{-1,0,1\}$ are simple.

Definition 2.4: The edges $e_{i j}$ take values in the fuzzy causal interval $[-1,1] . e_{i j}=0$ indicates no causality, $e_{i j}>0$ indicates causal increase in $C_{j}$ increases as $C_{i}$ increases (or $C_{j}$ Decreases as $C_{\mathrm{i}}$ Decreases). $E<0$ indicates causal decrease or negative causality. $\mathrm{C}$ decreases as $C$ increases (And or $C_{j}$ increases as $C_{i}$ decreases). Simple FCMs have edge values in $\{-1,0,1\}$. Then if causality occurs, it occurs to a maximal positive or negative degree. Simple FCMs provide a quick first approximation to an expert stand or printed causal knowledge. If increase (Or decrease) in one concept leads to increase (or decrease) in another, then we give the value 1.If there exists to relation between the two concepts, the value 0 is given. If increase (or decrease) in one concept decreases (or increases) another, then we gives the value -1 . Thus FCMs are described in this way. Consider the or concepts $C_{1} \ldots C_{n}$ of the FCM. Suppose the directed graph is drawn using edge weight $e_{i j} \in\{0,1,-1\}$. The matrix $\mathrm{E}$ be defined by $\mathrm{E}=\left(e_{i j}\right)$, Where the $e_{i j}$ is the weight of the directed edge $C_{i}, C_{j}$. E is called the adjacency matrix of the FCM, also known as the connection matrix of the FCM. It is important to note that all matrices associated with an FCM are always square matrices with diagonal entries as zero.

Definition 2.5: Let $C_{1}, C_{2} \ldots C_{n}$ be the nodes of an FCM. Let $A=\left(a_{1}, a_{2} \ldots a_{n}\right)$, where $a_{i} \in\{0,1\} . A$ is called the instantaneous state vector and it denoted the on off position of the node at an instant

$$
\begin{array}{ll}
a_{i}=0 & \text { if } a_{i} \text { is off }=1 \\
a_{i}=1 & \text { if } a_{i} \text { is on, where } i=1,2, \ldots, n .
\end{array}
$$

Definition 2.6: Let $C_{1}, C_{2} \ldots C_{n}$ be the nodes of an FCM. Let $C_{1} C_{2}, C_{2} C_{3} \ldots C_{i} C_{j}$, be the edges of the FCM $(\mathrm{i} \neq \mathrm{j})$. Then, the edges form a directed cycle. An FCM s said to be cyclic if it possesses a directed cycle. An FCM is said to be a cyclic if it does not possess any directed cycle.

Definition 2.7: An FCM with cycles is said to have a feedback.

Definition 2.8: Where there is a feedback in an FCM, i.e., When the causal relations flow through a cycle in a revolutionary way, The FCM is called a dynamical system.

Definition 2.9: Let $C_{l} C_{2}, C_{2} C_{3} \ldots C_{i} C_{j}$, be a cycle when $C_{i}$ is switched on and if the causality flows through the edges of a cycle and if it again causes $C_{i}$, We say that the dynamical system goes round and round. This is true for any node $C_{i}$, for $i=1,2 \ldots n$. The equilibrium state for this dynamical system is called the hidden pattern.

Definition 2.10: If the equilibrium state of a dynamical system is a unique state vector, then it is called a fixed point. Consider a FCM with $C_{l}, C_{2} \ldots C_{N}$ as nodes. For example let us start the dynamical system by switching on $C$. Let us assume that the FCM settles down with $\mathrm{C} 1$ and $C_{n}$ on, i.e. the state vector remains as $(1,0,0 \ldots 0,1)$. This state vector $(1,0$, $0 \ldots 0,1)$ is called the fixed point [4].

Definition 2.11: If the FCM settles down with a state vector repeating in the form $A_{1} \rightarrow A_{2} \rightarrow \ldots A_{\mathrm{I}} \rightarrow A_{1}$. Then this equilibrium is called limit cycle.
Definition 2.12: Finite number of FCMs can be combined together to produce the joint effect of all the FCMs. Let $E_{l}$, $E_{2} \ldots E_{p}$ be adjacency matrices of the FCMs with nodes $C_{l}$, $C_{2} \ldots C_{n}$. Then the combined FCM [5] is got by adding all the adjacency matrices $E_{1} \ldots E_{p}$. We denote the combined FCM adjacency matrix by $E=E_{1}+E_{2}+\ldots+E_{p}$.

Definition 2.13: Let $\mathrm{P}$ be the problem under investigation. Let $\left\{C_{1}, C_{2} \ldots C_{n}\right\}$ be n concepts associated with $\mathrm{p}$ (n very large). Now divide the number of concepts $\left\{C_{1}, C_{2} \ldots C_{n}\right\}$ into classes $S_{1} \ldots S_{t}$ where classes are such that

1) $S_{i} \cap S_{i+1} \neq \phi$ where $(i=1,2 \ldots t-1)$

2) $\cup s_{i}=\left(c_{1}, \ldots, c_{n}\right)$

3) $\left(s_{i}\right) \neq s_{j}$ if $i \neq j$ in general

Now we obtain the FCM associated with each of the classes $S_{1} \ldots S_{t}$. We determine the relational matrix associated with each $\mathrm{S}$. Using theses matrices we obtain a $\mathrm{n} \times \mathrm{n}$ matrix. This $\mathrm{n}$ $\times \mathrm{n}$ matrix is the matrix associated with the combined overlap block FCM (COBFCM) of blacks of same sizes.

Definition 2.14: Suppose $\mathrm{A}=\left(a_{1} \ldots a_{n}\right)$ is a vector which is passed into a dynamical system E. Then $\mathrm{AE}=\left(a_{1}^{\prime}, \ldots, a_{n}^{\prime}\right)$. After thresholding and updating the vectors suppose we get $\left(b_{1} \ldots b_{n}\right)$. We denote that by $\left(a_{1}^{\prime}, a_{2}^{\prime}, \ldots, a_{n}^{\prime}\right) \rightarrow\left(b_{1}, b_{2}, \ldots, b_{n}\right)$. Thus the symbol $\rightarrow$ means that the resultant vector has been thresholded and updated. FCMs have several advantages as well as some disadvantages. The main advantage of this method it is simple. It functions on experts opinions. When the data happens to be an unsupervised one the FCM comes handy. This is the only known fuzzy technique that gives the hidden pattern of the situation. As we have a very well known theory, which states that the strength of the data depends on the number of experts' opinions we can use combined FCMs with several experts' opinions. At the same time the disadvantage of the combined FCM is when the weightages are 1 and -1 for the same $C_{i} C_{j}$. We have the sum adding to zero thus at all times the connection matrices $E_{l} \ldots E k$ may not be comfortable for addition. This problem will be easily overcome if the FCM entries are only 0 and 1 .

\section{A Study on Problems Faced By the People With DISABILITIES (PWDS) USING COMBINED OVERLAP BLOCK FuZZY COGNITIVE MAPS (COBFCMS) [6]}

For that, using linguistic questionnaire and the expert's opinion we have taken the following eleven concepts $\left\{C_{l}, C_{2} \ldots\right.$ $\left.C_{11}\right\}$

The following concepts are taken as the main nodes for our problem.

$C_{1}$ - No proper healthcare

$C_{2}$ - Poor nutrition

$C_{3^{-}}$Improper clothing

$C_{4}$ - No proper shelter

$C_{5}$ - No recreation

$C_{6}$ - No proper school education

$C_{7}$ - No employment / self-employment

$C_{8}$ - No information about the SSHGs

$C_{9^{-}}$Welfare measures of government never reach the rural PWDs 
$C_{10^{-}}$Poor economy

$C_{11}$ - Marriage remains a question mark

From the survey and the interviews taken from the rural PWDs and the caretakers were the vital problems suffered by them.

\section{A. No Proper Healthcare}

They said their economic conditions forced them to neglect the PWDs from giving them proper medical care and or taking them for routine check up or even taking them to local doctor at time of emergency. For to take them out for medical aid one has to spend also on the conveyance which is an economic burden on the caretakers. Thus healthcare is one of the problems faced by the PWDs.

\section{B. Poor Nutrition}

Due to poor economic conditions or reasons best known to the caretakers it was very difficult for the caretakers as well as family members of the PWDs to give "good" nutritious food to the PWDs. Only the male breadwinner alone was in a position to get somewhat nutritious food. It was also observed that not only the PWDs but also the family members suffered from lack of nutrition.

\section{Improper Clothing}

When the very basic needs such as food and medical aid were under strain one cannot think of comfortable clothing for the disabled to comfort his / her disability. Thus clothes depending on the weather conditions cannot be provided by the caretakers to the dependent PWDs. Baring this as the PWDs are assumed not only as a burden but a curse on the family and a social stigma they were not given new cloths even during the festivals. Infact they were made to wear the old dresses available in the family of the caretakers. Also they were not given frequent changes of the dress, which we observed to be dirty and torn. Further this poor hygiene made them unhealthy and gave a distressed out look.

\section{No Proper Shelter}

The PWDs from the rural poor were mostly segregated from the family in the verandah or in some common places such as temples or in the back yard under a thatch for reasons best known to the caretakers. They were alone in most cases and found to be lonely, sad and depressed.

\section{E. No Recreation}

The sole recreation in villages as of today is watching TV (Television) or hearing music. As they (PWDs) happen to hold stigma they are never taken out for any public function, even for festivals to temples / churches or for recreation to cinemas, parks, picnic, tours or visits to relatives. They are made to be at home as watchman when the entire family leaves the house for a function or festival or recreation. Games / painting or indoor games etc can certainly be provided to them if at all they have better economy. For in rich society PWDs are given nice recreations.

\section{F. No Proper School Education}

When they are denied the basic needs the question of sending them to school under these circumstances can only be construed as a waste of time for the caretakers. That is why there were several PWDs who had not even entered the school. They would by no means be interested in giving a special training depending on the disability. Thus the skills, talents and the gifts in these economically poor disabled never come to light. Yet another major reason is both the caretakers and the PWDs felt it to be a disgrace as the disability in a family was considered to be a curse and so they suffered a social stigma.

\section{G. No Employment / Self-Employment}

The poor PWDs can be technically trained in certain jobs fetching skills depending on their deformity so that they can sustain themselves and lead a dignified life. But unfortunately this is absent in most of the cases. They are treated as if they are some condemned nonliving objects. The caretakers and the family fail to make use of them in a positive way.

\section{H. No Information about the SSHGs}

The information about Special Self-Help Groups (SSHG) and the structure and functional mechanism of SSHGs are unknown to PWDs. It never reaches them the reasons being that the rural PWDs are ignorant as uneducated and segregated. The caretakers never want the public or anyone to know about the presence of a PWD in their family as they view it as a social stigma for the marriages in these families will turn out to be a problem.

Further the PWD in their family is viewed as a curse so they tend to isolate them consequent to which the information about SSHG or benefits announced by government or their rights as PWDs or free medical aid etc is never known to them. So the question of utilizing these never occurs. Further even if the information is found in dailies or advertisements in TVs PWDs are ignorant of it as they do not have any access to them.

\section{Welfare Measures of Government Never Reach the Rural PWDs}

Welfare measures never reach the rural poor PWDs for the reasons said in above point.

\section{J. Poor Economy}

The economic condition as per the survey done by the NGO LAMP NET is that only $2.1 \%$ of the families with a PWD as its member have an income over Rs. 3000 per month. $75.9 \%$ of them get an income less than Rs. 1000 per month.

\section{K. Marriage Remains a Question Mark}

When the very basic needs are not given it has become a near impossibility for the caretakers to even think of marriage for the PWDs under their custody. This is evident from the survey data that in the reproductive age, they remain unmarried where as in the unproductive age the percentage of married PWDs is considerably larger than the percentage of married in the reproductive age.Another important reason is 'social stigma'. It is difficult to get them married off due to this major social hurdle. Poverty is also another reason for the PWD's remaining unmarried. Mainly these were the attributes given by the experts' asproblem of the PWDs. The group of experts was drawn from the PWD's caretakers / guardians / parents of PWDs and NGOs. 


\section{Method OF DETERMining HidDEN PATTERN}

Let $C_{1}, C_{2} \ldots C_{n}$ be the nodes of an FCM, with feedback. Let $\mathrm{E}$ be the associated adjacency matrix. Let us find the hidden pattern when $C_{l}$ is switched on. When an input is given as the vector $A_{1}=(1,0,0 \ldots 0)$, the data should pass through the relation matrix E. this is done by multiplying $A_{1}$ by the matrix E. Let $A_{1} E=\left(a_{1} \ldots a_{n}\right)$ with the threshold operation that is by replacing $a_{i}$ by 1 if $a_{i}>\mathrm{k}$ and $a_{i}$ by 0 if $a_{i}<k$ ( $k$ is a suitable positive integer). We update the resulting concept; the concept $C_{1}$ is included in the updated vector by making the first coordinate as 1 in the resulting vector. Suppose $A_{1} E \rightarrow$ $\mathrm{A}_{2}$ then consider $\mathrm{A}_{2} \mathrm{E}$ and repeat the same procedure. This procedure is repeated till we get a limit cycle or a fixed point.

\section{CONCEPT OF THE PROBLEM}

Using the linguistic questionnaire and the expert's opinion we have taken the following nine concepts $\left\{C_{1}, C_{2} \ldots C_{11}\right\}$

$C_{1}$ - No proper healthcare

$C_{2}$ - Poor nutrition

$C_{3}$ - Improper clothing

$C_{4}$ - No proper shelter

$C_{5}$ - No recreation

$C_{6}$ - No proper school education

$C_{7}$ - No employment / self-employment

$C_{8}$ - No information about the SSHGs

$C_{9^{-}}$Welfare measures of government never reach the rural PWDs

$C_{10}$ - Poor economy

$C_{11}$ - Marriage remains a question mark

Now we proceed on to apply the effect of combined overlap block. FCM of equal length. Let us consider the eleven concepts $\left\{C_{1}, C_{2} \ldots C_{11}\right\}$. We divide these concepts into cyclic way of classes, each having just four concepts in the following way. [7]

1) The directed graph and the relation matrix for the class $C$ $=\left\{C_{1}, C_{2}, C_{9}, C_{10}\right\}$. The expert opinion of an expatriate of care takers of PWDs is given as follows:

\section{Care takers of PWD:}

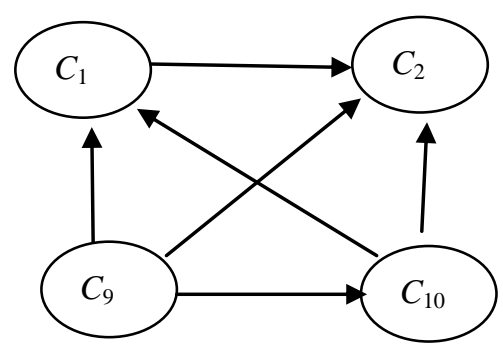

$$
C_{1}\left[\begin{array}{cccc}
C_{1} & C_{2} & C_{9} & C_{10} \\
C_{9} & 1 & 0 & 1 \\
0 & 0 & 0 & 0 \\
1 & 1 & 0 & 1 \\
1 & 1 & 0 & 0
\end{array}\right]
$$

2) The directed graph and the relational matrix for the class $\left.C=C_{1}, C_{2}, C_{3}, C_{8}\right\}$. Given by the expert is as follows: NGO leaders)

\section{NGO Leaders:}

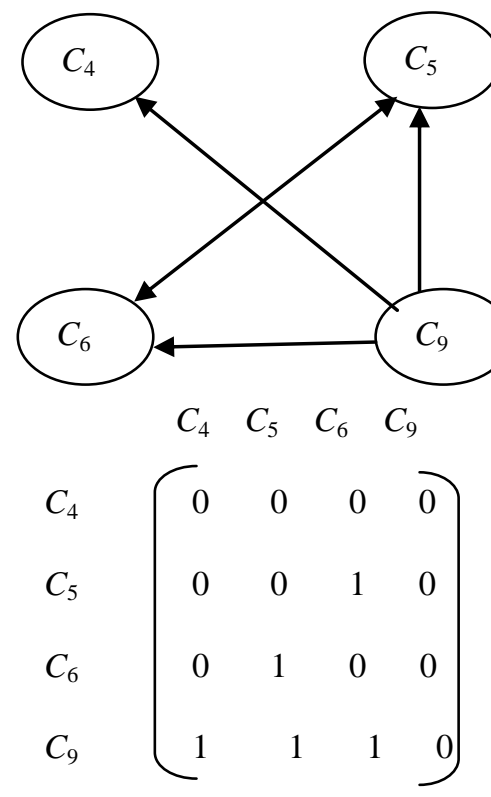

3) The directed graph and the relational matrix for the class $C=\left\{C_{3}, C_{7}, C_{8}, C_{11}\right\}$. Given by the expert is as follows: PWDs themselves,

\section{PWDS themselves:}

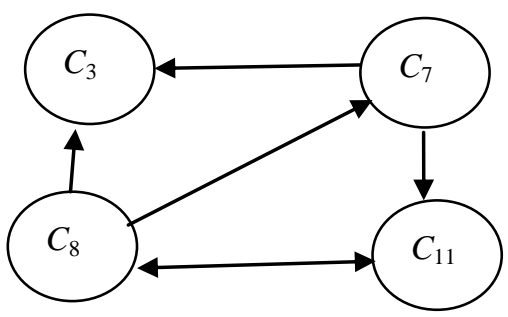

$$
\begin{gathered}
C_{3} \\
C_{7} \\
C_{8} \\
C_{11}
\end{gathered}\left[\begin{array}{cccc}
0 & C_{7} & C_{8} & C_{11} \\
1 & 0 & 0 & 0 \\
1 & 1 & 0 & 1 \\
0 & 0 & 1 & 0
\end{array}\right]
$$

4) The directed graph and the relation matrix for the class $C$ $=\left\{C_{1}, C_{3}, C_{8}, C_{9}\right\}$ given by the expert is as follows: woman self help leaders

5) The directed graph and the relation matrix for the class $C$ $=\left\{C_{1}, C_{2}, C_{7}, C_{9}\right\}$ given by the expert is as follows: special self help leaders

6) The combined direct graph and combined overlap block FCM of equal sizes as follows[8]

Woman self help leaders: 


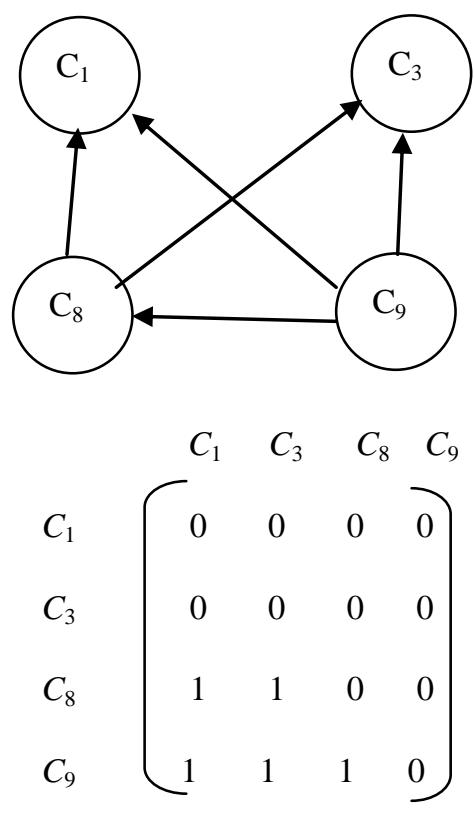

\section{Special self help leaders:}

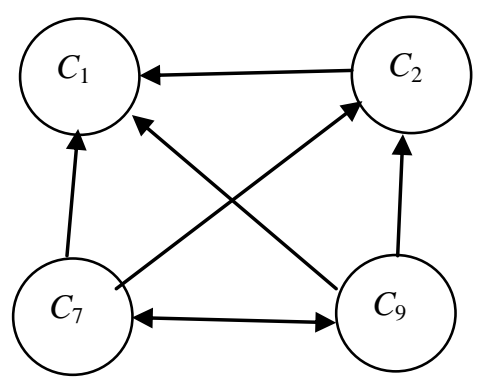

$$
\begin{aligned}
& C_{1} \\
& C_{2} \\
& C_{7} \\
& C_{9}
\end{aligned}\left[\begin{array}{llll}
C_{1} & C_{2} & C_{7} & C_{9} \\
0 & 0 & 1 & 0 \\
1 & 0 & 0 & 0 \\
0 & 1 & 0 & 1 \\
1 & 1 & 1 & 0
\end{array}\right]
$$

\section{Combined FCM:}

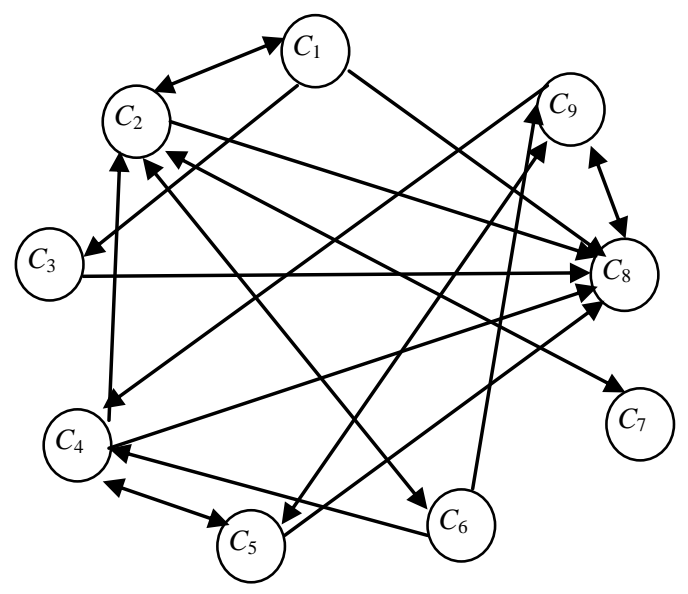

Now using the matrix A of the combined overlap block FCM, We determine the hidden pattern. Suppose the concept $\mathrm{S}_{1}$ is in the on state and another nodes are in the off sate. Let

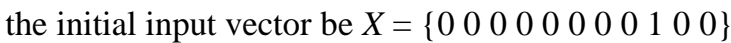

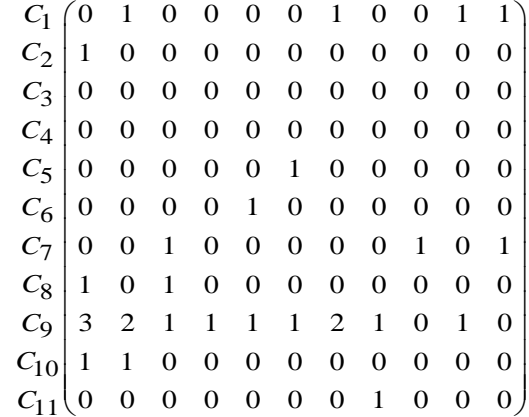

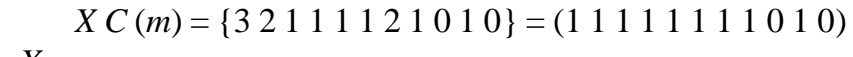

$$
\begin{aligned}
& X_{1} C(m)=\left\{\begin{array}{llllllllll}
3 & 2 & 2 & 0 & 1 & 1 & 1 & 1 & 0 & 1
\end{array}\right\} \\
& \rightarrow\left\{\begin{array}{llllllllll}
1 & 1 & 0 & 1 & 1 & 1 & 1 & 0 & 1 & 1
\end{array}\right\}=X_{2}
\end{aligned}
$$

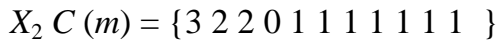

$$
\begin{aligned}
& \rightarrow(1110011111111\}=X_{3} \\
& X_{3} C(m)=\left\{\begin{array}{lllll}
64312232121\}
\end{array}\right. \\
& \rightarrow(111111111111\}=X_{4}
\end{aligned}
$$

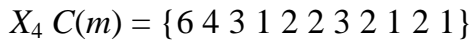

$$
\begin{aligned}
& \rightarrow(111111111111\})=X_{4}
\end{aligned}
$$

where $\rightarrow$ Denotes the resultant vector after thresholding and updating.

$X_{4}$ is the hidden pattern which is the fixed point. [9]

\section{CONCLUSION}

While analyzing FCM, When the concept $C_{9}$ "is in the on state, the other concepts $C_{1}, C_{2}, C_{3}, C_{4}, C_{5}, C_{6}, C_{7}, C_{8}, C_{10}, C_{11}$ are in the on state, because of Welfare measures of government never reach the rural PWDs there will be, $C_{1}$ - No proper healthcare, $C_{2}$-Poor nutrition, $C_{3}$-Improper clothing, $C_{4}$ - No proper shelter, $C_{5}$ - No recreation, $C_{6}$ - No proper school education, $C_{7^{-}}$No employment / self-employment, $C_{8^{-}}$No information about the SSHGs, $C_{10^{-}}$Poor economy, $C_{11^{-}}$ Marriage remains a question mark. Therefore Welfare measures of government never reach the rural PWDs is the major reason for people having Disabilities.

\section{SUGGESTIONS}

The study points out the fact the root cause of the problems with persons with disabilities is due to lack of sufficient welfare measures of the government to rehabilitate them. Person with Disabilities (equal opportunities, protection of rights and full participation) act 1995 continue to remain only in paper, in order to implement the provisions of the act national level. state level, district level, block level coordination committees must be formed to periodically review the proper implementation steps must be taken to raise awareness among the people and the PWDs on the basics of disabilities and on their rights to special care, right to food, clothing, shelter, education, health and employment . 


\section{FUTURE WORK}

Problems of different kinds of disability will be analysed by using fuzzy models.

\section{ACKNOWLEDGMENT}

The authors wish to thank director Mr. Ashok vergesh (Hindustan University -Chennai).

\section{REFERENCES}

[1] R. Axelrod, "Structure of decision: The congnitive maps of political elites," Princeton University, 1976

[2] W. B. Vasanthakandasamy and A. Victordevadoss, "Some new fuzzy techniques," Joural of inst.of Math \& Computer science.

[3] B. Kosko, "Fuzzy cognitive maps," International Journal of man-machine studies, January, 1986.

[4] B. Kosko, Neural Networks and Fuzzy System, Prentice Hall of India, 1997.

[5] B. Kosko, "Hidden patterns in Combined and adaptive Knowledge Networks," in Proc. International Conference of Neural Networks (ICNN-86)1988 377-393.

[6] W. B. V. Kandasamy, F. Smarandache, and A. P. Prakash, Mathematical Analysis of the Problems faced by the People with Disabilities, May 2012.

[7] A. Victordevadoss, Change of quality of life through literacy in Bhutan using Fuzzy cognitive mapping

[8] W. B. Vasantha Kandasamy and M. Ram Kishore Symptom, "Disease Model in Children using FCM," Ultrasci., vol. 11, 1999, pp. 318-324.

[9] W. R. Taber, "Fuzzy Cognitive Maps Model Social Systems," AI expert, vol. 9, pp.18-23, 1994

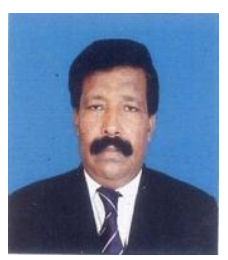

A. Praveen Prakash is a member of Research Promotion Committee and professor of Dept. Of Mathematics, Hindustan Institute of Science and Technology, Hindustan University, Padur, Chennai603.He has thirty six years of teaching experience and ten years of research experience. Currently four scholars are working for their Ph.D under his supervision and guidance. His area of specilisation is Fuzzy Logic and models. All through his life he has been involved in voluntary action for the cause of persons living under difficult circumstances such as the dalits,women,persons with disabilities and adolescents.He utilises Fuzzy models to anaslyse socio psychological problems encountered by such of those people in distress. He has authored two Books on High School Mathematics. His Ph.D thesis,'An Analysis of the Problems faced by Persons with Disabilities 'was published as Book in United State supervision experience.He has published 12 articles in National and International Journals.

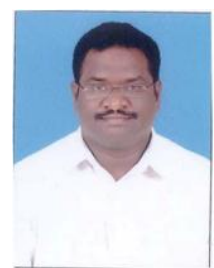

A. Rajkumar is a member of Research Promotion Committee and assistant professor of Department of Mathematics, Hindustan Institute of Science and Technology, HindustanUniversity, Padur, Chennai 603. He has Ten years of teaching experience and two years of research experience. Currently research scholars (Ph.D) under his supervision and guidance of victor devadoss.His area of specilisation is Fuzzy Logic and models. He has published 12 articles in International Journals.

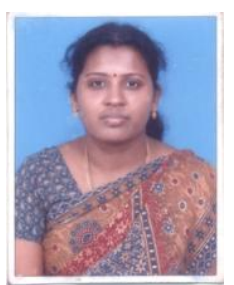

N. Jose Parvin Praveena is a member of Research Promotion Committee and assistant professor of Department of Mathematics, K.C.G. college of technology, He has seven years of teaching experience and two years of research experience. His area of specialization is Fuzzy Logic and models. He has published 8 articles in International Journals. under the guidance of Praveen prakash. 